\title{
A NEW NEUROSURGICAL TOOL INCORPORATING DIFFERENTIAL GEOMETRY AND CELLULAR AUTOMATA TECHNIQUES
}

\author{
ALEXANDER R. OSHMYANSKY \\ Centre for Mathematical Biology \\ University of Oxford \\ 24-29 St. Giles Oxford, OX1 3LZ, UK \\ E-mail: alexander.oshmyansky@stx.ox.ac.uk \\ PHILIP K. MAINI \\ Centre for Mathematical Biology \\ Oxford Centre for Integrative Systems Biology \\ University of Oxford \\ 24-29 St. Giles Oxford, OX1 3LZ, UK \\ E-mail: maini@maths.ox.ac.uk
}

\begin{abstract}
Using optical coherence imaging, it is possible to visualize seizure progression intraoperatively. However, it is difficult to pinpoint an exact epileptic focus. This is crucial in attempts to minimize the amount of resection necessary during surgical therapeutic interventions for epilepsy and is typically done approximately from visual inspection of optical coherence imaging stills. In this paper, we create an algorithm with the potential to pinpoint the source of a seizure from an optical coherence imaging still. To accomplish this, a grid is overlaid on optical coherence imaging stills. This then serves as a grid for a two-dimensional cellular automation. Each cell is associated with a Riemannian curvature tensor representing the curvature of the brain's surface in all directions for a cell. Cells which overlay portions of the image which show neurons that are firing are considered "depolarized".
\end{abstract}

Keywords: Cellular automata; neuronal dynamics; curved "cellular manifold".

\section{Seizure modelling}

There has been significant effort put into modelling seizure progression. A seizure is generally defined as a transient disturbance of cerebral functioning due to an abnormal paroxysmal neuronal discharge in the brain. ${ }^{1}$ The majority of effort has been focused on modelling results obtained from electroencephalograms (EEGs). Models have been created utilizing partial differential equation (PDE) techniques, ${ }^{2}$ stochastic differential equations, ${ }^{3}$ nonlinear dynamics models, ${ }^{4}$ and a variety of other methods. As a potential application of this work, an ideal model would prove an effective tool to supplement qualitative clinician assessment of EEG data for diagnostic purposes. Although this has not yet been achieved, significant progress has been made. 
In this paper we focus an modelling data on seizure progression obtained from optical coherance imaging. The end purpose of this research is for therapeutic rather than diagnostic use. During the process of optical coherence imaging, changes in the optical spectroscopic properties of neocortical tissue are directly visualized. ${ }^{5}$ The changes in spectroscopic properties are directly correlated to changes in cerebral hemodynamic properties such as blood oxygenation and blood volume. As cerebral hemodynamics can serve as a proxy for neuronal activity, as it does in functional magnetic resonance imaging, optical coherence imaging effectively results in visualization of neuronal activity on the brain surface.

A recent application of this technique has been to visualize seizures occurring intraoperatively. ${ }^{5}$ Epilepsy is a condition of recurrent seizures which often originate from a focus of pathologic neurons in the cerebral cortex. ${ }^{1}$ Epilepsy which is refractory to medical therapy is often treated by surgical resection of epileptic foci. However, it is often difficult to locate an epileptic focus for resection. This often results in much more tissue than is necessary being removed during therapeutic surgery (a common epilepsy surgery is temporal lobectomy in which the entire temporal lobe of a patient is removed when it is believed an epileptic focus is present on the lobe) or too little being removed which eliminates the therapeutic benefit of surgical intervention. Optical coherence imaging allows direct qualitative inspection of seizure initiation and progression from an epileptic focus, thus allowing better identification of the boundaries of foci.

The aim of this paper is to model seizure progression on the surface of the brain with a simple wave model on top of a curved manifold. It is hypothesized that this model can be directly applied to intra-operative optical coherence imaging data to effectively predict in a more quantitative fashion the origin of a seizure and the boundaries of an epileptic focus. This would then allow more effective resection within epilepsy surgery.

\section{Combining Geometry with Cellular Automata}

In creating a model that would be most effective intra-operatively, one had to be created that would allow for easy selection of initial conditions. Essentially, we want to eventually allow surgeons to take an image obtained through imaging of intrinsic optical signals (IIOS, another term for optical coherence imaging) and select easily yet still accurately which groups of neurons are activated on the image. Ideally, an algorithm based on our model would then determine the boundaries of an epileptic focus.

To select groups of activated neurons on an image, we propose overlaying a grid on an image obtained by IIOS. Cells which are partially filled are then labelled as "depolarized." It should then be easy to run a cellular automata ${ }^{6}$ simulation on the grid after creating rules for how seizure progression should behave. In fact, there is already an extensive literature on simulating neuronal activity using cellular automata $^{7}$ though usually not in so direct a fashion. 
However, the surface of the brain is of course not flat and therefore a flat grid would obviously be ineffective for modelling the cerebral or cerebellar surface without some modification. The modification we propose is to designate each cell in the simulation with a "curvature" given by a Riemannian curvature tensor.

The concept of curvature on a Riemannian manifold is abstract but remarkably versatile. As a brief review, a manifold is a space such that an open neighborhood around each point in the space is locally Euclidean (further information on Riemannian geometry can be found in Petersen ${ }^{8}$ ). A Riemannian manifold more specifically is a manifold with a defined inner product at each point. The Riemannian curvature tensor can be used to define the curvature of a Riemannian manifold. If $u$ and $v$ are coordinate vector fields and $w$ is a manifold, the Riemannian curvature tensor can be defined by:

$$
R(u, v) w=\nabla_{u} \nabla_{v} w-\nabla_{v} \nabla_{u} w
$$

where $\nabla$ is the covariant derivative, a generalization of the derivative which gives a derivative along the tangent vectors of a manifold. Effectively, the tensor thus gives all information about the curvature of a point in a single quantity which does not rely on local coordinates.

Being able to refer to curvature with a single quantity is quite useful for our purposes. Background independence (lack of dependence on a local coordinate system) also proves to have utility. By defining a curvature at each cell in a cellular automata simulation, one can approximate the curvature of a given manifold. Rules of a cellular automata simulation can then be easily modified to determine geodesic motion on a curved "cellular manifold" rather than on a flat cellular automata grid.

\section{Using Cellular Manifolds to Model Seizures}

From observation of images obtained by IIOS and basic knowledge of neural physiology, ${ }^{9}$ we will create rules for how a depolarized cell should interact with other cells on a flat grid. In order to mimic neuronal dynamics grossly, all cells surrounding a depolarized cell should become depolarized at the next time step after a wave of depolarization has had time to fully propagate through a cell. After a given time period, the depolarized cell should begin to "repolarize" and enter a refractory period during which it cannot be depolarized.

It reality, some portions of a propagating depolarization wavefront on the cortex would of course move faster than others given individual axon and dendrite lengths and connectivity of individual neurons. However, at the level of coarsity that is being dealt with in any given grid cell, thousands of neurons are being represented simultaneously. We feel that it is safe to ignore this effect as it should prove minimal at the coarsity described.

The following rules are thus proposed for the cellular automata that shall be run:

(1) At each step all squares in contact with a depolarized square become depolarized if $\left|\nabla_{u} v\right| * t \leq c$. $\nabla_{u}$ is the covariant derivative in the direction of 
vector $u$. The vector $u$ is a unit vector in the direction the depolarization from the original depolarized square. $v$ is a tangent vector to the brain manifold at a touching square. $t$ is the total time in terms of time steps that has been spent at a square. $c$ is a constant given by the speed of the propagation of the wavefront.

(2) If a square depolarizes its neighboring squares, it becomes repolarized.

(3) A repolarized square cannot be depolarized again for $t_{r}$ time steps, given by a the neuronal refractory period.

Note that all vectors $v$ are given by the Riemann curvature tensor at a given point. Using the definition of the Riemann curvature tensor in local coordinates $x^{\mu}$ (written in Einstein summation notation):

$$
R_{\sigma \mu \nu}^{\rho}=d x^{\rho}\left(R\left(\partial_{\mu}, \partial_{\nu}\right) \partial_{\sigma}\right)
$$

the tangent vectors $v$ are merely the coordinate vector fields $\partial_{\mu}=\partial / \partial x^{\mu}$ while vectors $u$ correspond with the coordinates $x^{\rho}$ that we choose based on the direction of depolarization wavefront propagation.

\section{Example and Qualitative Comparison to Actual Data}

Data for direct comparison to simulations is not yet available to the authors at this time. In lieu of this, we show a simplified example of the proposed technique and show images obtained by IIOS during a seizure near the same area from available literature $^{10}$ for comparison. The similarities in depolarization progression seen on visual inspection are clearly observed.

To illustrate the proposed technique, we overlay a grid on a plain intraoperative photograph and run a cellular automata with the proposed rules starting from an arbitrary cell deemed to be the epileptic focus. Image 1 is taken at the very beginning of the Sylvian fissure which is underneath the middle cerebral artery which extends from the bottom left to the center of the image. For the sake of simplicity in illustration, curvature will be calculated only at the Sylvian fissure and only unit curvature values will be used. (That is, $\nabla_{u} v$ will always equal either 1 or -1$)$.

For the simulation, cells filled in yellow on image 2 have $\nabla_{u} v=1$ and cells filled in blue have $\nabla_{u} v=-1$. On image 3, the actual simulation is shown. Green cells are depolarized in at each time-step.

An actual sequence from optical coherence imaging of a seizure is shown in image 4. The similarity to the pattern of spread of the depolarization wavefront can be qualitatively observed. The spread pattern is very similar to that predicted from the simplified simulation. Plans for more direct quantitative analysis of simulations are discussed in the next section. 


\section{Future work}

Given the methodology proposed in the paper, a practical neurosurgical tool will ultimately be developed. Ideally, this tool will calculate brain curvature from preoperative scans (MRI or CT) for each individual patient. Surgeons will be able to select areas seen to be depolarized on images taken by IIOS and the tool will calculate the borders of epileptic foci.

Present work is focusing on creating a generic phantom which may be used for the majority of patients without necessitating access to imaging. Once a phantom is complete, further work will compare directly modelled seizures with those actually visualized. This will directly assess the quality of the proposed algorithm.

There is also a significant amount of interesting mathematics that can likely be extracted from the concept of a cellular manifold. Work without direct intent of an application will be done to further expand on this idea.

\section{Figures}

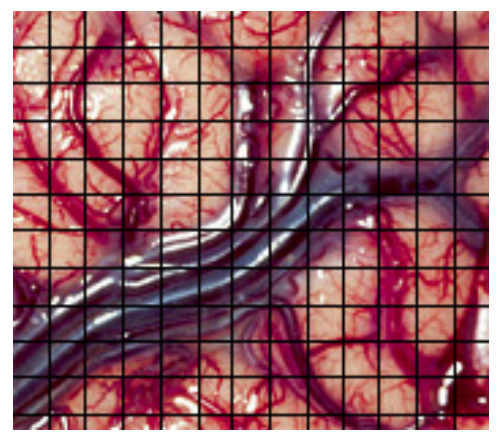

Fig. 1. Intraoperative picture of the beginning of the Sylvian Fissure. Image by Robert F. Spetzler, M.D., Barrow Neurological Institute, Phoenix, AZ, USA.

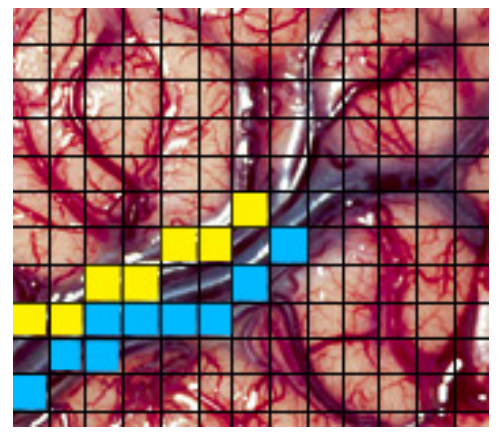

Fig. 2. Highlighted Squares represent curvature of brain. At white (yellow) squares $\nabla_{u} v=1$ and at gray (blue) squares $\nabla_{u} v=-1$. 
Time Step

1
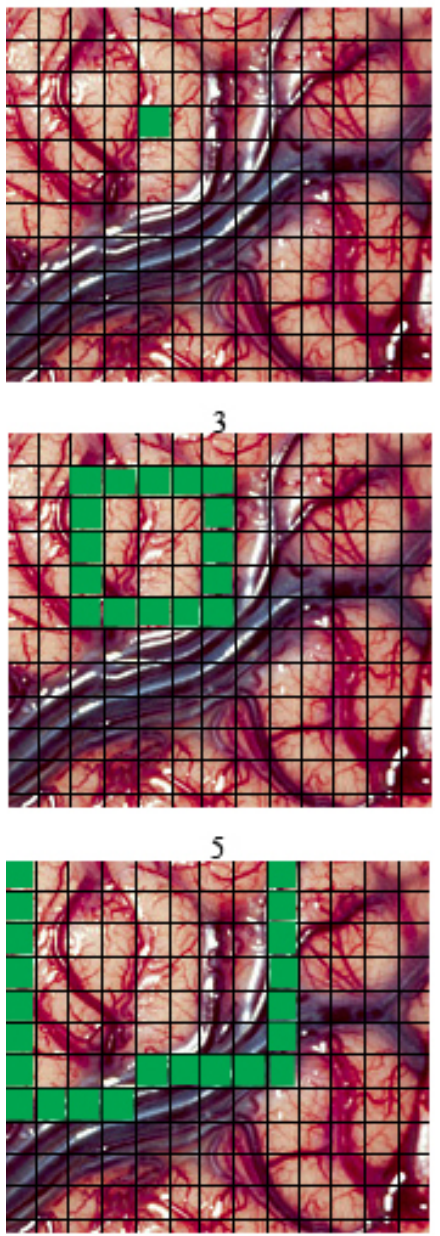

2

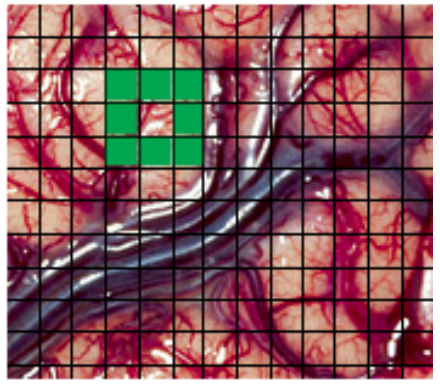

4
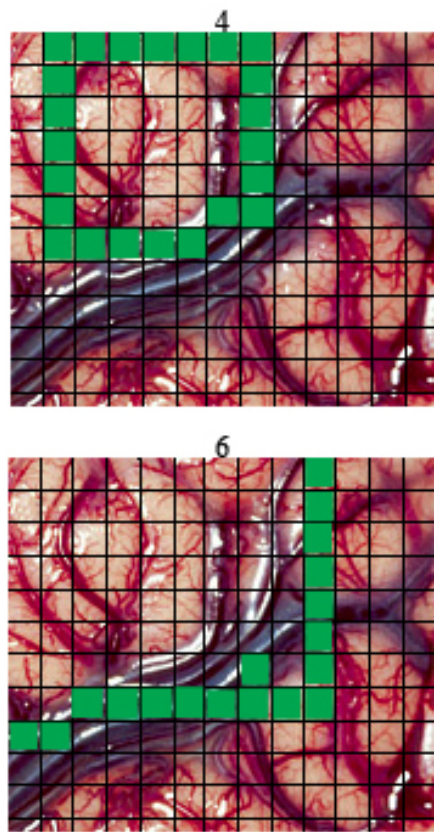

Fig. 3. Cellular automata simulation of seizure progression. Cells filled gray (green) are depolarized. 

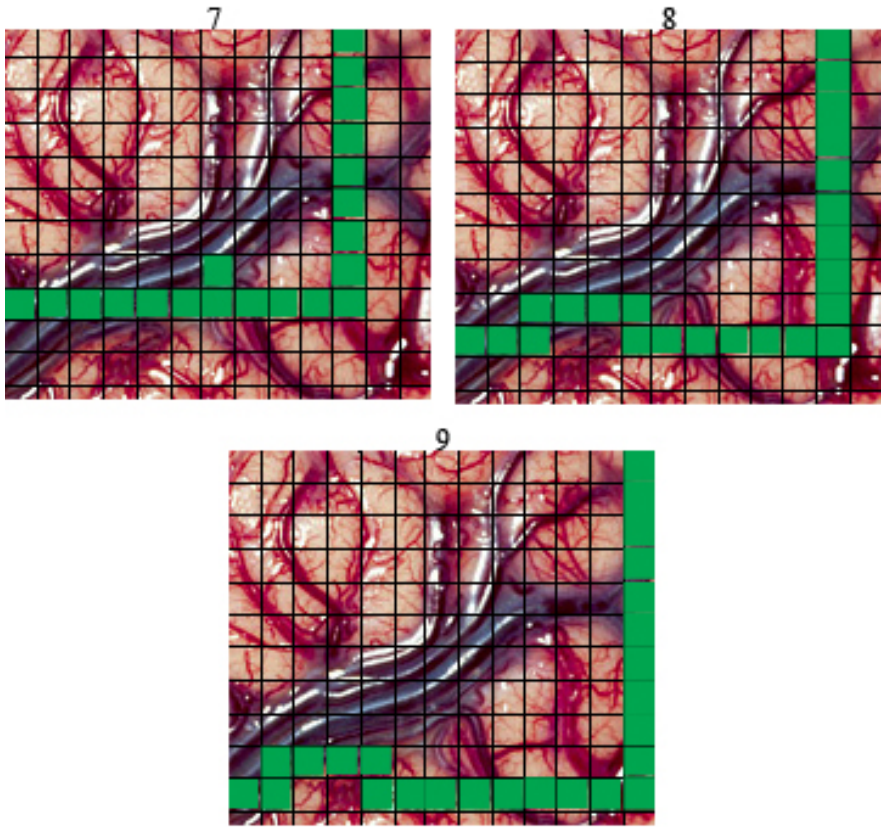

Fig. 3. (Continued)

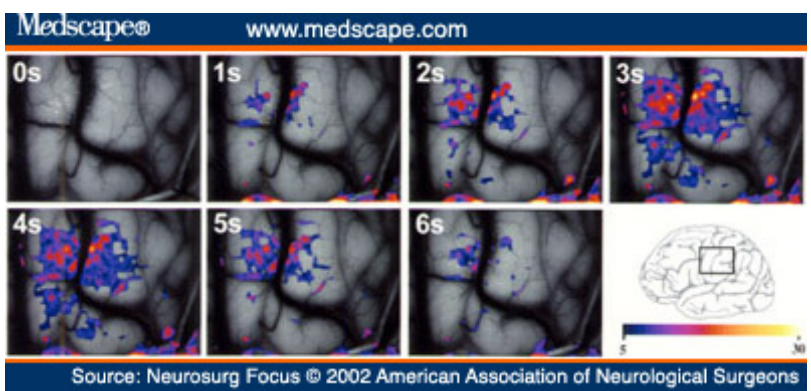

Fig. 4. Optical imaging stills from a seizure triggered near the same area as the simulation was run in. Not the similar spread pattern to the simulation up to $4 \mathrm{sec}$. at which point the seizure begins to resolve. Image from Ref. 10.

\section{Acknowledgments}

The authors would like to thank the Marshall Aid Commemoration Commission for their support of this research. We would also like to thank the students and staff of the Oxford Centre for Mathematical Biology for their input and insight. Additionally, we would like to the thank Dr. Michael Haglund of the Duke University 
Neurosurgical Department for demonstrating the utilization of IIOS during epilepsy surgery and for his support.

\section{References}

1. L. M. Tierney, Jr., S. J. McPhee, M. A. Papdakis, Current Medical Diagnosis and Treatment, Lange (2004).

2. M. Bertalmio, L. T. Cheng, S. Osher and G. Sapiro, Journal of Coputational Physics 174(2), 759 (2001).

3. T. D. Frank and P. J. Beek, Phys. Rev. E 264, 021917 (2001).

4. C. J. Stam, Clin. Neurophysiol. 116(10), 2266 (2005).

5. M. M. Haglund and D. W. Hochman, Epilepsia 45, 43 (2004).

6. S. Wolfram, Cellular Automata and Complexity. Westview Press (2002).

7. L. O. Chua, L. Yang, IEEE Transactions on Circuits and Systems 37(10) 1257 (1988).

8. P. Petersen, Riemannian Geometry, Springer (2006).

9. D. Purves, G. J. Augustine, D. Fitzpatrick, and W. C. Hall, Neuroscience, Sinauer Associates (2004).

10. N. Pouratian, A. F. Cannestra, N. A. Martin, A. W. Toga, Neurosurg Focus, 13(4) (2002). Available at http://www.medscape.com/viewarticle/443946_2. 